\title{
CITTÀ DI CARTA E TERREMOTI. LICONOGRAFIA DELLA CATASTROFE IN BASILICATA (1851-1857)
}

Lia Romano

Università degli Studi di Napoli Federico II

ORCID: https://orcid.org/0000-0002-1854-1652

\section{SOMMARIO}

Il contributo intende analizzare la ricca documentazione iconografica relativa a due importanti terremoti che hanno colpito la "Provincia di Basilicata" nell'Italia meridionale nel 1851 e nel 1857. In entrambi i casi i danni furono accuratamente rappresentati da disegni, e per il sisma del 1857 anche da fotografie, fonti preziose per la comprensione e l'analisi dei danni inferti al patrimonio urbano e architettonico della regione. Gli elaborati grafici redatti da artisti, architetti e dall'ingegnere-geologo Robert Mallet, seppur realizzati con finalità differenti, restituiscono all'unisono un'interessante spaccato della Basilicata di metà Ottocento caratterizzato da un paesaggio urbano frutto delle trasformazioni prodotte dai frequenti disastri naturali. L'analisi e il confronto critico di tale documentazione iconografica e delle descrizioni che la accompagnano costituisce un utile strumento per l'analisi dei caratteri costruttivi della regione nonché per lo studio e l'interpretazione delle lente modificazioni e delle tracce lasciate dal sisma sull'identità dei piccoli centri urbani.

Parole chiave: terremoto 1851, terremoto 1857, Basilicata, Vulture, Robert Mallet

\section{ABSTRACT}

This paper aims to analyse the extensive iconographic documentation relating to two major earthquakes that struck the province of Basilicata, in southern Italy, in 1851 and 1857. In both cases the damage was accurately represented in drawings, which provide valuable sources for understanding and analysing the region's urban and architectural heritage. The graphic works created by artists, architects and the engineer-geologist Robert Mallet introduce a fascinating cross-section of mid-19th-century Basilicata, which was characterised by an urban landscape shaped by frequent natural disasters and the transformations they brought about. The analysis and comparison of this iconographic documentation and descriptions are useful tools in the study of the region's constructions, the interpretation of the slow pace of change, and the impact that earthquakes had on the identity of small towns.

Keywords: 1851 earthquake, 1857 earthquake, Basilicata, Vulture, Robert Mallet

\section{Due catastrofi all'alba dell'Unità d'Italia}

I terremoti che colpirono la "Provincia di Basilicata" nell'agosto del 1851 e nel dicembre del 1857 furono gli ultimi due grandi sismi della storia del Regno delle Due Sicilie'.

Il governo borbonico aveva già affrontato numerose altre catastrofi coordinando le fasi emer- genziali e le ricostruzioni e ottenendo, come nel caso del terremoto calabro del 1783, anche una grande riconoscibilità a livello europeo per la gestione del post-evento calamitoso e per l'impegno profuso dai più importanti eruditi del tempo nella pubblicazione di testi scientifici di grande livello. Basti citare l'Istoria de' fenomeni del tremuoto av- 
venuto nelle Calabrie, e nel Valdemone nell'anno 1783 posta in luce dalla Reale Accademia delle Scienze e delle Belle Arti di Napoli, che con il suo poderoso apparato letterario e iconografico, aveva fotografato lo stato di desolata distruzione del paesaggio e dei centri urbani calabri all'indomani del terremoto ${ }^{2}$. A ciò va aggiunta la redazione del primo regolamento antisismico della storia frutto dell'intenso lavoro degli ingegneri Francesco La Vega e Antonio Winspeare unitamente al Vicario generale Francesco Pignatelli, inviato nei territori danneggiati appena dopo la catastrofe con il ruolo di gestire la fase d'emergenza e guidare l'avvio della ricostruzione ${ }^{3}$.

L'esperienza maturata a seguito del sisma di fine Settecento -che distrusse completamente oltre cinquanta centri urbani, di cui trentadue riedificati in altro sito- rappresentò una base di fondamentale importanza per la definizione dei primi provvedimenti a seguito del sisma che colpì nel 1805 la "Provincia di Molise". Tuttavia, appare chiaro come la politica attuata in Calabria Ultra rappresentò in questo caso un riferimento solo nella fase emergenziale: infatti, se per entrambi i contesti (Calabria e Contado di Molise) vennero nominati due uomini attivi sulla scena politica con poteri assoluti per i soccorsi immediati, gli ingegneri militari che li accompagnarono furono inviati con ben diversi obiettivi. Nel caso calabro, Francesco Pignatelli di Strongoli, Vicario Generale delle Calabrie, lavorò con Antonio Winspeare e Francesco La Vega, incaricati di designare le aree per la fondazione delle città completamente distrutte che dovevano essere riedificate in altro sito. In Molise, invece, sembrò del tutto assente, poiché probabilmente non necessario, la volontà di spostare i centri urbani maggiormente danneggiati: ai tecnici inviati, guidati da Luigi Marchese, regio ingegnere camerale, venne richiesto di redigere perizie relative ai danni del patrimonio architettonico e di indicare interventi atti a evitare ulteriori crolli come puntellamenti ed eventuali demolizioni. L'obiettivo, dunque, a dispetto di una simile organizzazione, sembrò essere fin dall'inizio completamente diverso, come anche, va detto, la situazione politica e amministrativa. II terremoto del 1805, infatti, colpì il Molise pochi mesi prima dell'invasione francese del Regno di Napoli e il governo, nonostante gli sforzi e le attività intraprese, non riuscì a portare a termine gli obiettivi preposti. La difficile situazione politica ed economica del Regno non permise di gestire la fase post-catastrofe come era stato fatto appena venti anni prima in Calabria ${ }^{4}$

Un clima politico particolarmente teso e reso ancora più complesso a seguito dei moti risorgimentali del 1848 caratterizzò gli ultimi anni del Regno delle Due Sicilie prima dell'Unità d'Italia. La difficile situazione economica della maggior parte delle regioni del meridione della penisola italiana contribuì ad alimentare e accrescere il malcontento della popolazione nei confronti della casa regnantes.

Il terremoto del 14 agosto del 1851 non fece che aggravare tale condizione ${ }^{6}$. II sisma oltre a danneggiare fortemente i centri urbani della regione del Vulture (corrispondente alla parte nord-orientale dell'attuale Basilicata), fu accompagnato anche da un lungo periodo di siccità seguito da violente alluvioni che comportarono grandi perdite della produzione agricola.

Il successivo sisma del 16 dicembre 1857, con epicentro in Val d'Agri, colpì un territorio ancora più esteso, compresi alcuni centri urbani già parzialmente distrutti dal terremoto del 1851. Un'immensa catastrofe naturale, definita dal Times di Londra il terzo sisma più forte della storia, avvenuto, come nel caso del 1805, all'alba di un grande mutamento politico e amministrativo: I'Unità d'Italia'.

In entrambi i casi il governo borbonico si dimostrò apparentemente celere nei provvedimenti. L'obiettivo, infatti, era quello di apparire forte sul piano internazionale e, per quanto possibile, riguadagnare la fiducia e il favore della popolazione. La stampa e la promozione di opere che descrivessero il ruolo dei Reali nella gestione della catastrofe, furono gli strumenti impiegati dal governo per dimostrare al Regno e a tutti gli Stati esteri il grande interessamento della casa regnante e la capacità di gestione degli eventi calamitosi. Tuttavia, se a seguito del sisma del 1851 le cronache e le "rappresentazioni" promosse ${ }^{8}$ riuscirono nel loro intento, dopo il terremoto del 1857, nonostante gli articoli pubblicati sul Giornale delle Due Sicilie' ${ }^{9}$, prevalse il senso di malcontento da parte della popolazione colpita, come testimonia sia l'opera del pastore limosino protestante Jean 
Louis Théophile Roller ${ }^{10}$ sia alcuni passi dell'innovativa pubblicazione di Robert Mallet" ${ }^{1}$.

Tali opere, alcune commissionate per motivi di propaganda politica o, come nel caso di Mallet, per puro interesse scientifico, furono corredate da una ricca documentazione iconografica che appare di grande interesse per l'analisi dei territori colpiti sia alla scala urbana sia a quella architettonica. In particolare l'opera di Robert Mallet descrisse i centri urbani dell'area del Vulture colpiti da entrambi i sismi, fornendo informazioni anche sull'avvio della ricostruzione post 1851 .

\section{Il terremoto di Melfi del 1851 e le rappre- sentazioni di Fergola, Flauti e Palma}

Dopo il sisma del 14 agosto 1851, il Re Ferdinando II visitò personalmente i centri colpiti e promosse la formazione di commissioni speciali per la raccolta, l'amministrazione dei fondi e la creazione dei consigli edilizi preposti al coordinamento della ricostruzione. La visita del Re fu prontamente immortalata nell'opera del paesaggista Nicola Palizzi' ${ }^{12}$ che rappresentò due vedute della città di Melfi, il centro maggiormente colpito, prima e durante la visita di Ferdinando II e del suo seguito, avvenuta nel settembre del $1851^{13}$. L'obiettivo di mostrare quanto il monarca fosse vicino ai suoi sudditi in un momento di tale difficoltà fu alla base anche delle opere di Salvatore Fergola, artista che aveva già in passato avuto numerose commissioni dai Borbone, in qualità di vero e proprio reporter grafico delle politiche reali ${ }^{14}$. II Re, infatti, venne rappresentato da entrambi gli artisti come un uomo misericordioso venuto ad aiutare il suo popolo. Le opere di Palizzi e Fergola non raffigurano la catastrofe o i danni ma l'evento connesso alla visita del monarca: si trattò, dunque, di una vera e propria propaganda politica condotta attraverso lo strumento dell'iconografia.

Accanto a tali vedute si collocano le rappresentazioni elaborate dall'architetto Achille Flauti e dall'ingegnere Francesco Palma. A seguito del sisma, infatti, furono inviati sui luoghi della catastrofe alcuni studiosi per effettuare sopralluoghi e fornire informazioni sui danni e sulle condizioni geologiche del territorio. II Flauti e il Palma accompagnarono tali eruditi per realizzare il corredo iconografico da accompagnare ai testi che sarebbero stati pubblicati una volta tornati a Napoli.

Luigi Palmieri, docente di logica e metafisica, e Arcangelo Scacchi, geologo e vulcanologo alla Regia Università di Napoli, furono inviati nei paesi colpiti dal sisma per conto della Reale Accademia delle Scienze di Napoli per condurre delle osservazioni di carattere geologico ${ }^{15}$. Si trattò della stessa Accademia che dopo il sisma calabro del 1783 aveva commissionato la già citata Istoria. I due studiosi, tuttavia, nonostante i continui rimandi al terremoto settecentesco, decisero di realizzare un'opera con un'impostazione differente, incentrata interamente sulle scienze naturali. Per questo motivo l'apparato iconografico venne ridotto al minimo e furono pubblicati solo i disegni ritenuti necessari a mostrare i danni inferti ai territori colpiti.

L'opera è divisa in tre parti: la prima è dedicata alla storia geologica della regione, la seconda riguarda il terremoto e la descrizione dei danni mentre la terza espone osservazioni sulla meteorologia elettrica.

La seconda parte appare di particolare interesse perché gli autori, oltre a fornire dati sulle condizioni topografiche dei centri colpiti si soffermarono sulle caratteristiche degli edifici, cercando di spiegare le motivazioni alla base dei diffusi crolli. Va evidenziato che tali osservazioni lasciano presupporre il coinvolgimento dell'architetto Achille Flauti che probabilmente non lavorò solo ai disegni ma collaborò attivamente anche alle ricerche dei due studiosi.

Palmieri e Scacchi riconobbero nella pessima muratura composta da conci di lava porosa non squadrati e legati con malta di pessima qualità la causa primaria dei cedimenti strutturali degli edifici. I leganti composti da una mistura di acqua, inerti e pochissima calce tendevano a polverizzarsi pochi anni dopo la costruzione, rendendo fortemente vulnerabili le architetture. Gli edifici costruiti a regola d'arte, con base larga e pochi piani, invece, riuscirono a resistere meglio, consentendo almeno agli abitanti di fuggire prima del crollo. Come possibili rimedi contro la forza funesta dei terremoti proposero, sulla scorta di un'indagine visiva condotta sugli edifici meno danneggiati, di utilizzare catene in ferro per rinforzare le murature, di costruire fondazioni soli- 


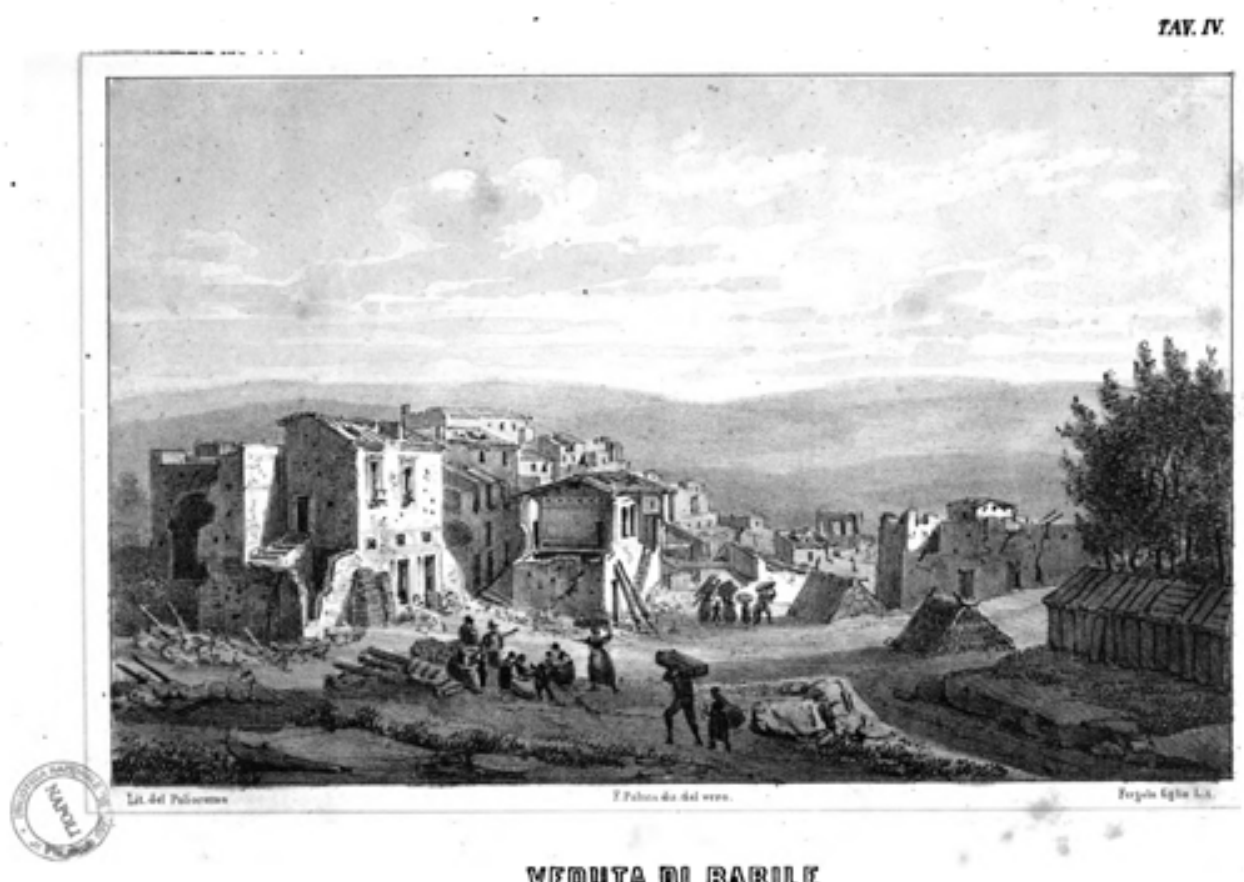

Fig. 1. Francesco Palma (disegnatore), Veduta di Barile (Paci, Giacomo. 1853. Relazione dei Tremuoti di Basilicata de 1851, Napoli: Stab. Tipogr. Ministero dell'Interno, tav. IV)

de nonché contrafforti, utili, come nel caso del Monastero di San Michele a Monticchio, a contenere i danni ${ }^{16}$. Misero in guardia anche sull'uso delle volte, generalmente molto sottili, formate anch'esse in conci di pietra lavica e non ben ammorsate alle partizioni verticali.

Alla denuncia dei pessimi caratteri costruttivi degli edifici accompagnarono la descrizione dei danni dei principali centri colpiti: Melfi, Rapolla, Barile, Rionero. In tutti i casi i centri avevano subito danni consistenti con crolli delle coperture e dissesti significativi delle murature.

Il testo è corredato da un apparato iconografico composto da sette tavole (realizzate da Achille Flauti): la prima è una cartografia dell'area colpita, la seconda una carta geologica del Vulture, la terza e la quarta delle vedute del vulcano spento, la quinta una veduta della città di Melfi, la sesta rappresenta la facciata della cattedrale di Rapolla e la settima riporta gli strumenti usati dagli studiosi per le osservazioni sull'elettricità atmosferica.
L'anno seguente, ovvero nel 1853 venne pubblicata un'altra descrizione del sisma a cura del fisico Giacomo Paci. Quest'ultimo fu inviato nella regione del Vulture per conto del Real Istituto di Incoraggiamento alle Scienze Naturali di Napoli e redasse una cronaca degli eventi e dei soccorsi attivati dai Borbone, principalmente con finalità di propaganda politica. L'impostazione, dunque, fu diversa da quella proposta da Scacchi e Palmieri e ciò risulta immediatamente comprensibile dall'indice ${ }^{17}$.

L'opera, anche in questo caso, è divisa in tre parti. La prima sezione è incentrata sugli effetti del sisma sui vari centri urbani, la seconda sulle istituzioni e i soccorsi mentre la terza è dedicata a una breve descrizione dei terremoti settecenteschi avvenuti in Calabria. Nella prima parte I'autore riportò una descrizione puntuale di ogni paese della regione del Vulture, soffermandosi brevemente sulla storia e sui monumenti più importanti. 


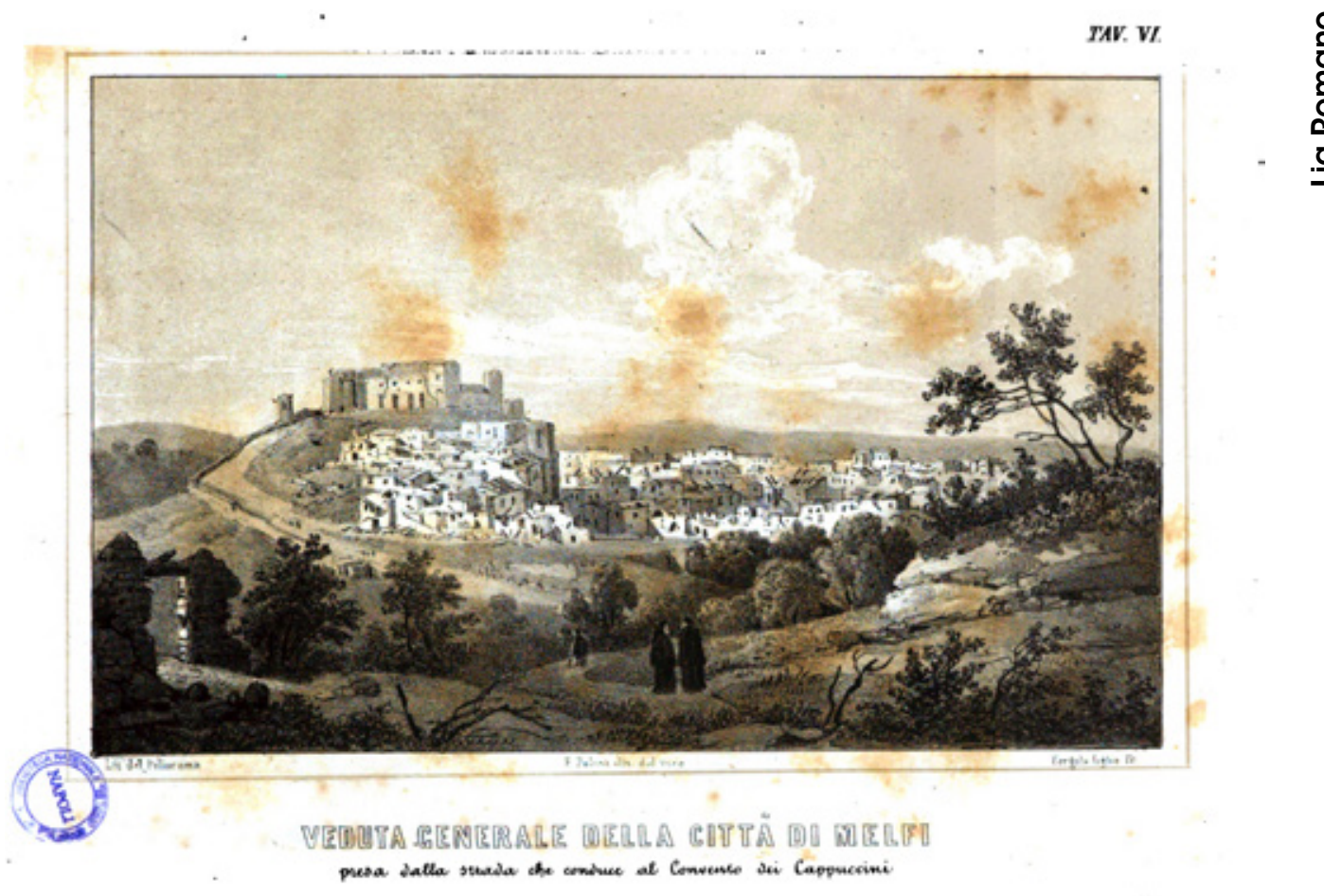

Fig. 2. Francesco Palma (disegnatore), Veduta generale della città di Melfi presa dalla strada che conduce al convento dei Cappuccini (Paci, Giacomo. 1853. Relazione dei Tremuoti di Basilicata de 1851, Napoli: Stab. Tipogr. Ministero dell'Interno, tav. VI)

Come Palmieri e Scacchi, Paci fornì informazioni sui caratteri costruttivi degli edifici evidenziando l'importanza dell'uso delle catene di ferro con funzione di concatenamento al fine di limitare i danni sulle fabbriche. A tal proposito, nel lavoro sono presenti anche riferimenti al terremoto del Molise del 1805 e alla memoria di Nicola Fergola, Delle concussioni e de' tremuoti, presentata all'Accademia Reale delle Scienze dal professore Flauti nella tornata del 23 gennaio $1852^{18}$.

Paci si servì della collaborazione dell'ingegnere Francesco Palma per la documentazione iconografica costituita da otto tavole inserite alla fine del testo. Fatta eccezione per la prima tavola, che rappresenta una pianta del distretto di Melfi, le altre sette sono vedute paesaggistiche (la seconda e la terza) nonché ritratti di città e di monumenti danneggiati (rispettivamente Barile, la cattedrale di Rapolla, Melfi, la chiesa di Sant'Agostino e la cattedrale di Melfi) (figg. 1-4).
La tavola dedicata alla cattedrale di Rapolla risulta particolarmente interessante perché si tratta di un monumento rappresentato non solo dal Palma ma anche da Fergola e dall'architetto Achille Flauti' $^{19}$ (figg. 5-7). Un confronto tra i diversi disegni appare molto utile per l'analisi dei danni e dei caratteri costruttivi della fabbrica. Paci e Fergola ritrassero la chiesa nel suo contesto -che fa da sfondo alla piazza circondata da edifici danneggiati- in cui un ruolo di primo piano è giocato dalla popolazione in procinto di pregare e di sgombrare le macerie. II punto di vista è ampio e la cattedrale non è che una parte della rappresentazione. Nella tavola del Flauti, invece, la facciata della chiesa, parzialmente tagliata sul lato sinistro, è l'unico elemento raffigurato in quanto alcuno spazio è concesso al contesto. L'autore si soffermò con meticolosità sul disegno delle singole pietre e sui dissesti. Da tutti e tre i disegni è chiaramente visibile la struttura della volta dell'abside, costituita da conci di pietra (probabilmente lavica) e la struttura lignea della copertura. II rivestimento della facciata 


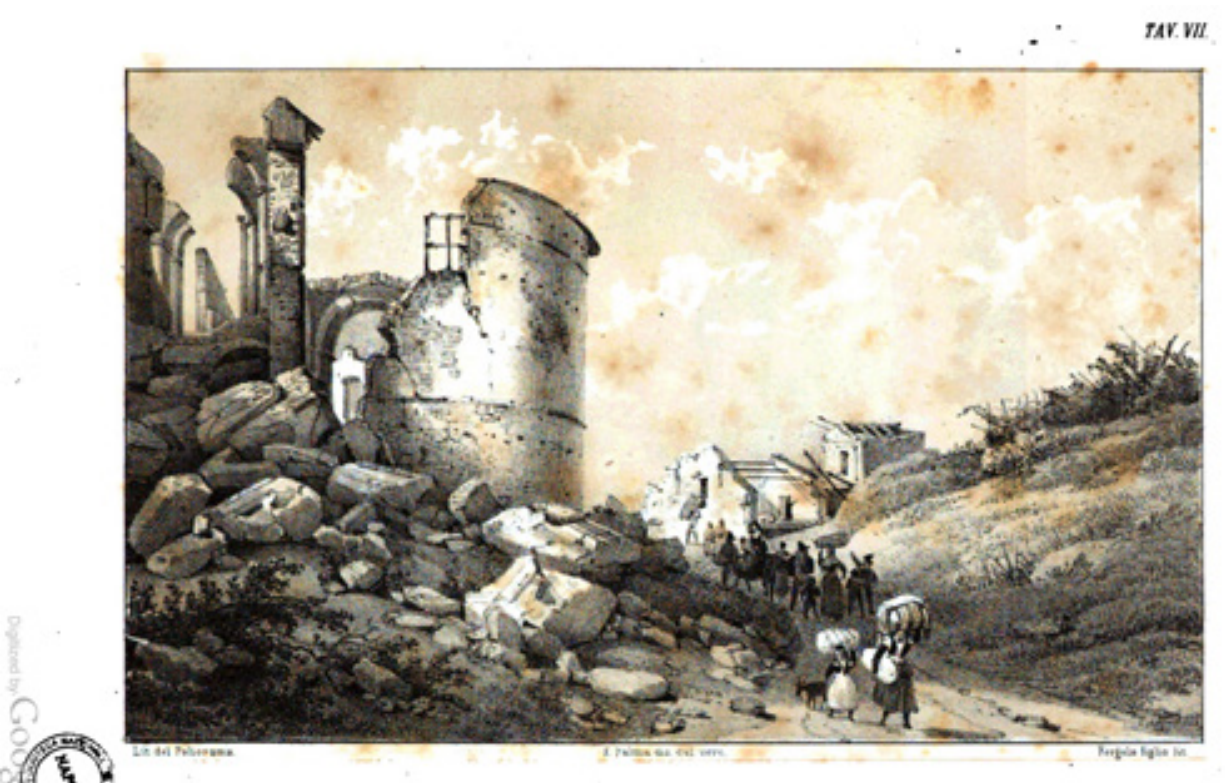

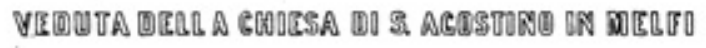

Fig. 3. Francesco Palma (disegnatore), Veduta della chiesa di Sant'Agostino in Melfi (Paci, Giacomo. 1853. Relazione dei Tremuoti di Basilicata de 1851, Napoli: Stab. Tipogr. Ministero dell'Interno, tav. VII)

in pietra ben squadrata, rinforzato da contrafforti, risulta parzialmente crollato, lasciando intravedere la tessitura sottostante e il gran numero di buche pontaie. Dal disegno di Flauti, sulla parte destra della facciata appare riconoscibile una soluzione di continuità verticale, che potrebbe corrispondere a un possibile "addossamento" di una parte dell'edificio nonché un'apertura a ogiva tamponata e trasformata in un piccolo vano rettangolare. Grande attenzione viene dedicata anche al disegno del portale di accesso alla chiesa e alle mancanze del rivestimento. Tutti e tre i disegni, sebbene leggermente diversi trattandosi di rilievi fatti dal vivo ${ }^{20}$ rappresentano una fonte di primaria importanza per la comprensione del manufatto nei suoi aspetti architettonici e costruttivi e per un confronto con i restauri condotti dopo il sisma nonché con la situazione attuale.

\section{Il terremoto del 16 dicembre 1857 nell'o- pera di Robert Mallet}

II sisma del 16 dicembre 1857 è noto per essere stato il primo terremoto "fotografato" della storia. I centri urbani colpiti, infatti, furono ripresi dal fotografo francese Alphonse Bernoud ${ }^{21}$ e da un altro reporter, noto come Grillier, assoldato dall'ingegnere-geologo irlandese Robert Mallet per realizzare alcune vedute da includere nell'opera sui cui stava lavorando22.

Quest'ultimo fu inviato nell'area colpita dal sisma dalla Royal Society di Londra con I'obiettivo di verificare sul campo le sue teorie sui fenomeni sismici. In particolare intendeva applicare un nuovo metodo di osservazione basato sull'analisi dei fenomeni e dei loro effetti per individuare l'epicentro del terremoto e la direzione dell'onda sismica23.

Il tecnico, aggiornato rispetto alle ricerche degli studiosi del Regno delle Due Sicilie, era consapevole che nessuno prima di lui avesse tentato di utilizzare questo metodo, compresi Palmieri e Scacchi di cui aveva letto l'opera e che aveva avuto l'occasione di conoscere personalmente ${ }^{24}$. I lavori dei suoi contemporanei non misero a punto nessuna nuova metodologia di indagine, limitandosi a raccontare la "storia" del sisma25. 

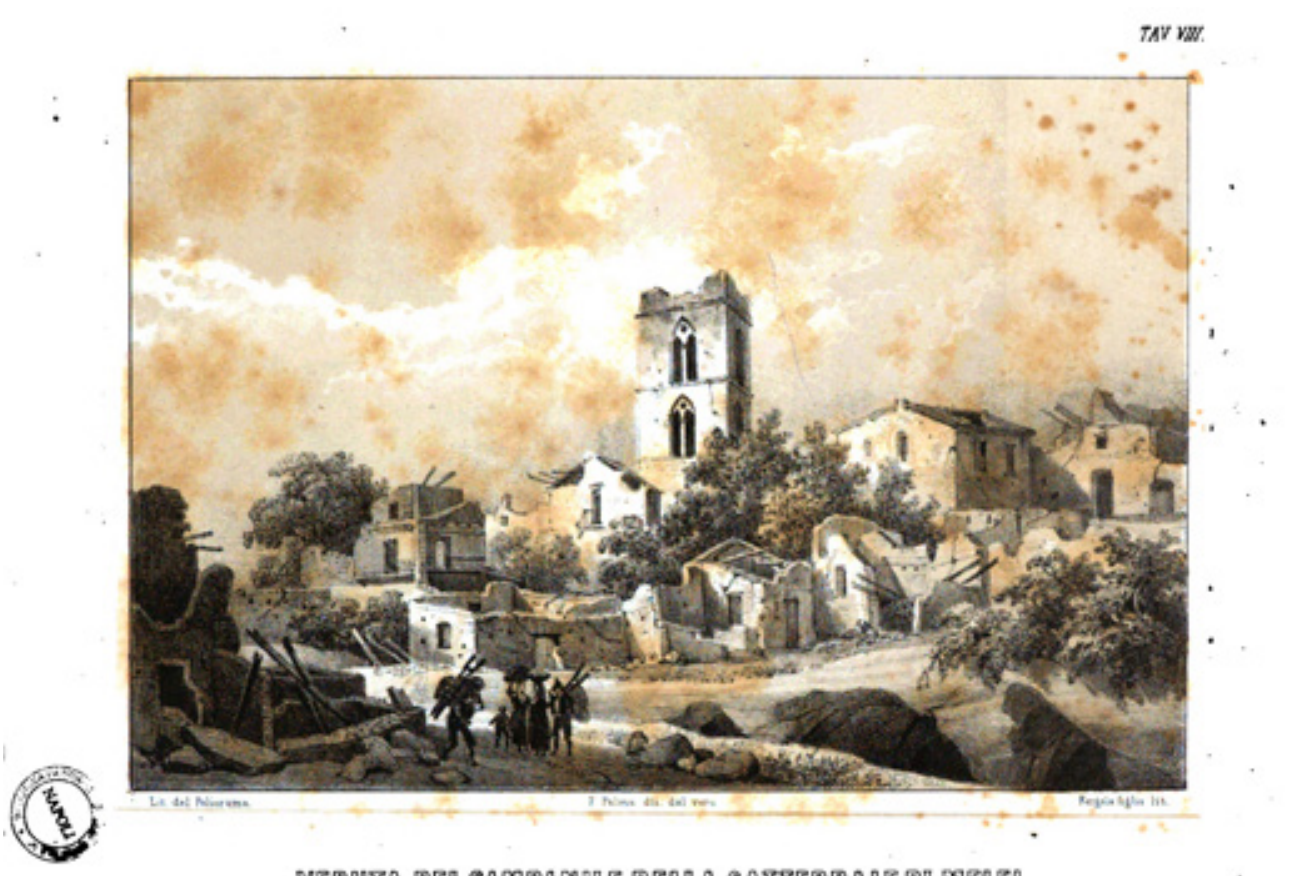

Fig. 4. Francesco Palma (disegnatore), Veduta del campanile della cattedrale di Melfi (Paci, Giacomo. 1853. Relazione dei Tremuoti di Basilicata de 1851, Napoli: Stab. Tipogr. Ministero dell'Interno, tav. VIII)

Seguendo la sua teoria, attraverso l'analisi degli effetti transitori del terremoto, sarebbe stato possibile dedurre informazioni sull'epicentro mentre grazie allo studio dei danni sugli edifici avrebbe potuto determinare la forza e il potere dell'onda sismica. Prima della messa a punto del suo metodo gli effetti dei terremoti erano stati descritti in maniera dispersiva, senza una logica che li riducesse in categorie. Era più che consapevole che la sua metodologia sarebbe diventata un potente strumento per le scoperte dei decenni successivi.

Robert Mallet fu una figura a tutto tondo in grado di muoversi con padronanza tra diverse scienze, dall'ingegneria alla fisica, dalla geologia all'architettura. Fu per questo motivo che nella sua opera, edita nel 1862, riuscì a dedicarsi con il medesimo approfondimento disciplinare alle varie tematiche inerenti il sisma e a corredare il testo con un ricchissimo apparato iconografico. Articolò il lavoro in tre sezioni dedicate rispettivamente alle domande di ricerca e al metodo, alla descrizione delle osservazioni effettuate in situ, alla classificazione dei dati e alle conclusioni.

Riconobbe l'insieme delle condizioni al contorno in grado di influenzare gli effetti dell'onda sismica sulle costruzioni come la forma, il tipo di fondazioni, di murature, i materiali costituenti la struttura e la disposizione delle aperture. Ma la sua attenzione non fu rivolta solo agli aspetti architettonici. Le descrizioni e i disegni dei centri urbani arroccati della Basilicata rappresentano oggigiorno documenti di grandissimo valore quali testimonianze della consistenza fisica dei piccoli agglomerati urbani in cui ancora oggi è possibile riconoscere i tratti caratteristici nonostante i danni provocati dai sismi del XX secolo ${ }^{26}$. Ad esempio, nel caso del piccolo paese di Petina, vicino Auletta (attuale provincia di Salerno) descrisse la felice condizione orografica del borgo che, situato su una terrazza di solido calcare lungo le pendici di una collina, era uscito illeso dalla scossa (fig. 8). $O$ il caso del centro di Sarconi per il quale redasse un'interessante sezione geologica e paesaggistica 


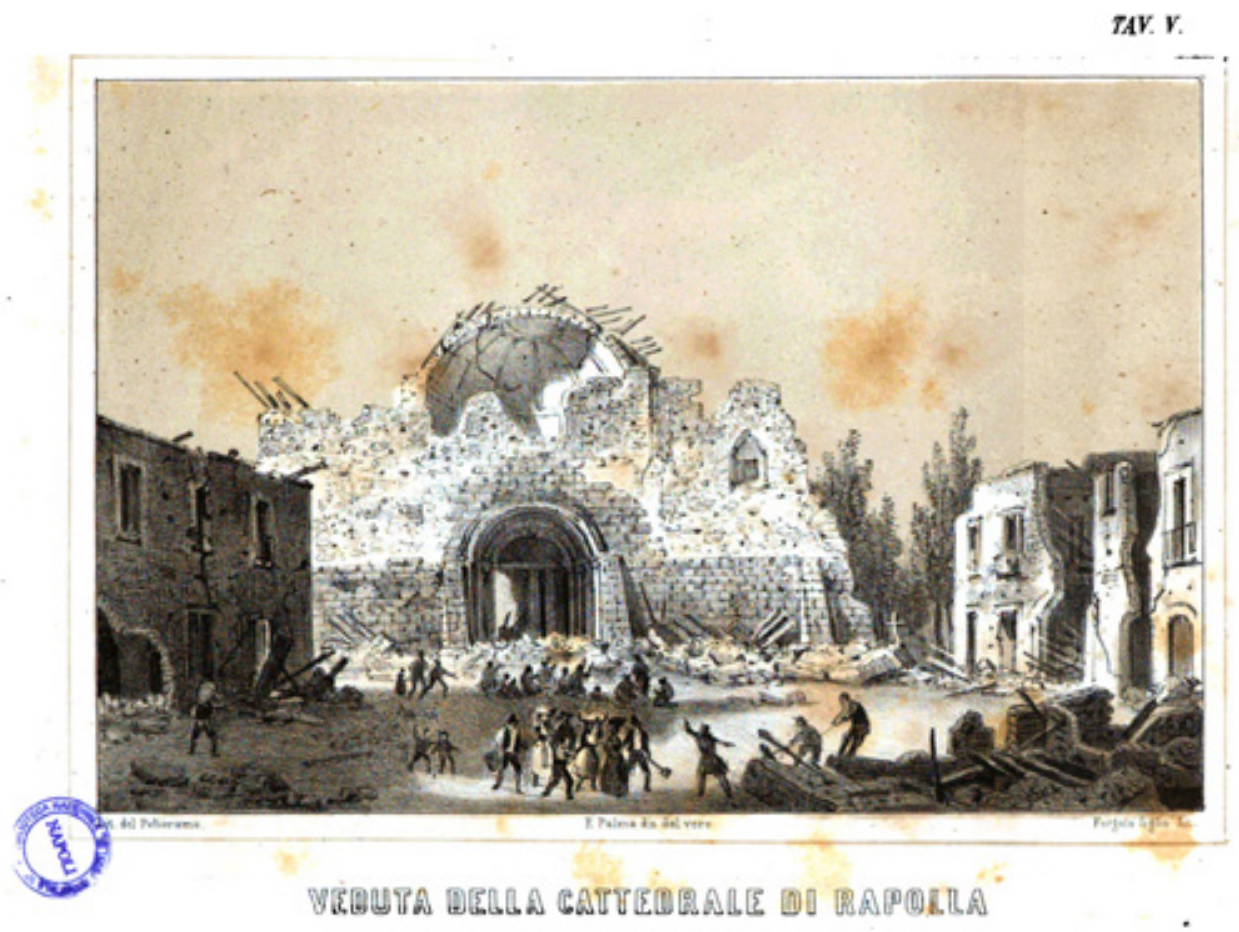

Fig. 5. Francesco Palma (disegnatore), Veduta della cattedrale di Rapolla (Paci, Giacomo. 1853. Relazione dei Tremuoti di Basilicata de 1851, Napoli: Stab. Tipogr. Ministero dell'Interno, tav. V)

con corrispondente descrizione del terreno (fig. 9). Il paese, ritenuto molto antico, era situato su un'alta e ripida scarpata di materiale alluvionale e di argilla sovrastante la riva di un fiume di grande ampiezza ${ }^{27}$. I disegni e le fotografie a corredo del testo sono un utilissimo strumento di studio per molteplici campi disciplinari, dalla storia dell'arte alla storia sismica.

Nel capitolo III della prima parte descrisse minuziosamente le tecniche costruttive dei centri della Val d'Agri, fornendo un quadro dettagliato dei materiali in uso. È possibile apprendere in tal modo che a differenza della regione del Vulture dove la pietra lavica risultava essere il materiale da costruzione più utilizzato in ragione della disponibilità in loco, nel centro della Basilicata oltre alla pietra calcarea, le strutture potevano essere realizzate anche in laterizio o in tufo locale. Evidenziò come la maggior parte delle volte delle chiese fosse costruita in laterizi pieni mentre quelle dei palazzi residenziali in tubi di terra cotta ${ }^{28}$.
Come Palmieri e Scacchi, denunciò le pessime modalità costruttive, concausa dei grandi danni sofferti dagli edifici, i quali se ben costruiti, sarebbero riusciti a sopportare meglio la forza dell'onda sismica.

Fece osservazioni sui differenti "tempi di vibrazione" dei materiali mettendo in guardia sulla grande vulnerabilità delle strutture asimmetriche perché disposte a lesionarsi in maniera differenziata. Anche gli effetti connessi alla flessibilità e all'elasticità degli edifici furono considerati così come il ruolo delle catene in ferro in grado di migliorare la resistenza della muratura. Fornì, a tal proposito, I'esempio del campanile di Atena (Lucana) che, dotato di un sistema di 4 catene a ogni livello, uscì completamente illeso dalla scossa, nonostante il resto del paese avesse subito gravissimi danni29. Rimarcò, inoltre, come il ferro fosse un materiale poco utilizzato a causa del suo elevato costo. Scrisse, infatti, che quello a disposizione veniva importato dall'estero o pro- 


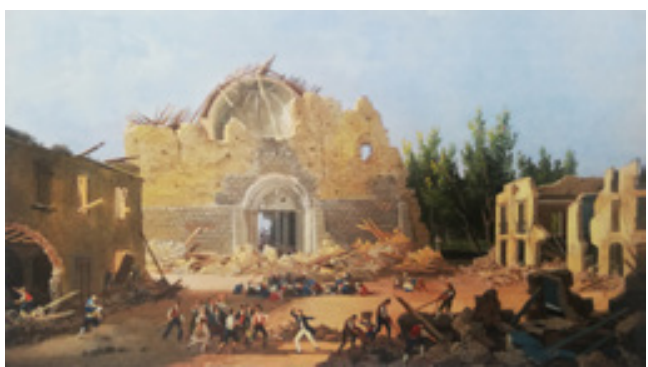

Fig. 6. Salvatore Fergola, La cattedrale di Rapolla parzialmente crollata nel terremoto del 1851 (Napoli, Museo Nazionale di San Martino) (Mazzocca Fernando, Martorelli Luisa and Antonio E. Denunzio (eds.). 2016. Fergola. Lo splendore di un regno. Catalogo della mostra (Napoli, 2 dicembre 2016/2 dicembre 2017). Venezia: Marsilio, 20, 183)

dotto nel Regno con un procedimento catalano obsoleto che non ne garantiva un livello di produzione adeguata alla richiesta. A suo parere, se gli edifici fossero stati ben costruiti non sarebbero crollati completamente, come invece era accaduto in molti casi.

Prestò grandissima attenzione all'andamento del quadro fessurativo e delle lesioni che, come sottolineò, normalmente seguivano l'andamento dei corsi di malta e solo in casi rari provocavano la rottura dei conci di pietra. Evidenziò come la forza di coesione della malta variasse in base ai materiali costituenti la miscela, al grado di umidità della stessa e dell'aria: notò infatti come i leganti molto secchi fossero più vulnerabili e soggetti a polverizzazione (figg. 10-11).

Introdusse il concetto, sebbene ancora non perfettamente definito, di edificio "in aggregato", spiegando come il danno dipendesse anche dalla direzione dell'onda sismica e dalla disposizione planimetrica dell'edificio sul territorio. In tal modo chiarì come mai fabbriche simili e adiacenti potessero essere danneggiate in maniera differente.

Terminò la prima parte del volume con una descrizione geologica dell'area colpita evidenziando come tutti i paesi fossero fondati su roccia o su depositi di argilla alluvionale e allegò in appendice la relazione di Palmieri e Scacchi. Sebbene definita ancora incompleta e con grandi lacune, a suo parere rappresentava il miglior rapporto redatto fino a quel momento sull'area di indagine.

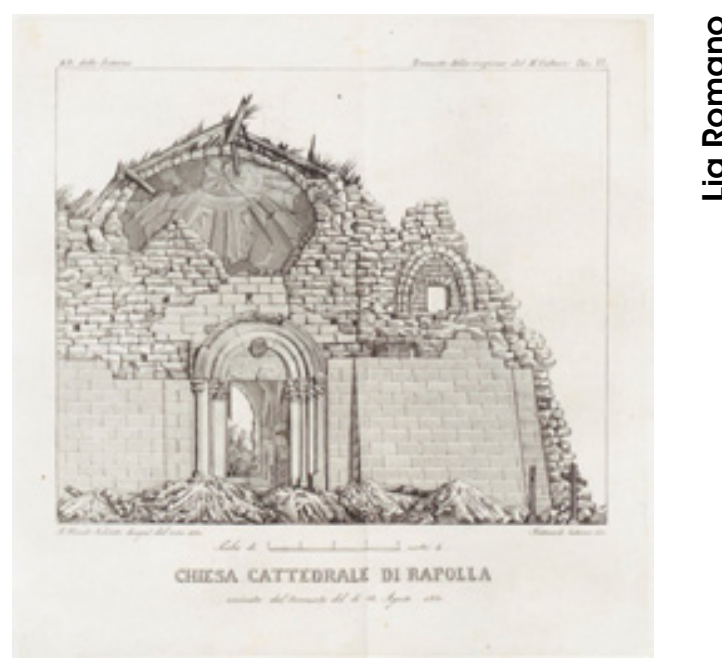

Fig. 7. Achille Flauti (disegnatore), Ferdinando Cataneo (incisore), Chiesa cattedrale di Rapolla rovinata dal tremuoto dei di' 14 agosto 1851 (Palmieri, Luigi and Scacchi Arcangelo. 1852. Della regione vulcanica del monte Vulture e del terremoto ivi avvenuto nel dì 14 agosto 1851. Napoli: G. Nobile, tav. VI)

Aprì il secondo volume con la storia sismica della regione e I'analisi delle modificazioni permanenti del livello del terreno e della costa. Dal capitolo IV iniziò la descrizione del viaggio. Se nella prima parte si limitò a delineare il suo metodo, nella seconda sezione lo applicò a tutti gli edifici esaminati. È in tal modo che il tecnico non solo fornì dettagliatissime informazioni sullo stato di conservazione dei centri e delle architetture ma individuò su casi concreti i principali fenomeni di dissesto causati generalmente dai terremoti e sistematizzati solo dai più recenti studiso. La rotazione fuori piano del timpano delle chiese, I'espulsione dei cantonali, la formazione di lesioni a taglio, il ribaltamento delle facciate furono solo alcuni dei tanti meccanismi individuati e descritti dal poliedrico ingegnere.

A sostegno dell'originalità del suo metodo citiamo la significativa descrizione del campanile e della cattedrale di Potenza, fortemente danneggiata dalla scossa ${ }^{31}$. Per quanto riguarda la torre campanaria, come nel caso di Atena, lodò il ruolo del sistema delle catene di ferro inserite a ogni livello della costruzione in pietra, eretta appena pochi anni prima e che probabilmente, anche per questa ragione, era riuscita a contrastare la forza delle onde sismiche. 


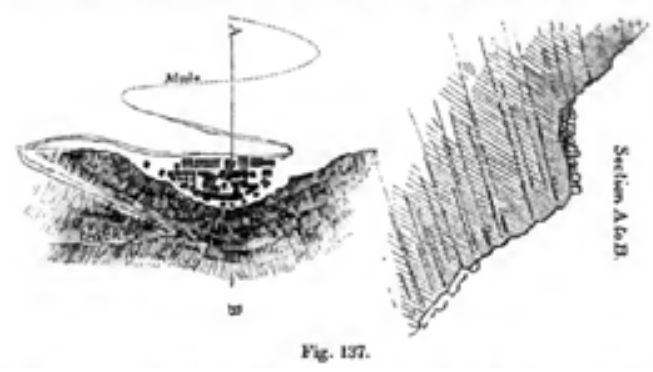

Fig. 8. Robert Mallet, Pianta e sezione del borgo di Petina vicino Auletta (Mallet, Robert. 1862. Great Neapolitan earthquake of 1857. The first principles of observational seismology as developed in the report to the Royal Society of London of the expedition made by command of the Society into the interior of the Kingdom of Naples, to investigate the circumstances of the great earthquake of December 1857. London: Chapman and Hall, t.1, 262, fig. 137)

Ben diversa sorte, invece, era toccata alla cattedrale, definita un edificio piuttosto moderno in stile "dorico-romanico" già restaurato a seguito di precedenti terremoti. Secondo Mallet, le murature e le volte erano costituite interamente da laterizi di buona qualità e apparivano fortemente lesionate. Nel corso dell'analisi dell'edificio e degli schizzi che stava realizzando ebbe modo di conoscere l'architetto del capitolo Giuseppe d'Errico, definito uomo di grande cultura nonché ottimo osservatore. Da quest'ultimo ricevette dei rilievi dettagliatissimi del quadro fessurativo dell'edificio consistenti in una sezione e nell'ipografia della struttura.

Una lunga fessura interessava tutta la volta della navata principale e la partizione verticale posta sul versante meridionale, in procinto di ribaltarsi. La cupola presentava profonde lesioni che avevano messo in luce la regolare tessitura della struttura in mattoni.

Mallet usò i disegni e le osservazioni di d'Errico per confermare le sue ipotesi sulla direzione dell'onda sismica. Nel capitolo successivo, inoltre, riportò una relazione dell'architetto sul calcolo del momento della scossa in cui il tecnico, a sostegno delle idee di Mallet, citò il considerevole ruolo delle catene in ferro nel miglioramento delle performance degli edifici in caso di terremoto ${ }^{32}$. Tali momenti di confronto dimostrano la capacità dell'ingegnere-geologo irlandese di relazionarsi con la comunità locale, nonostante le difficoltà

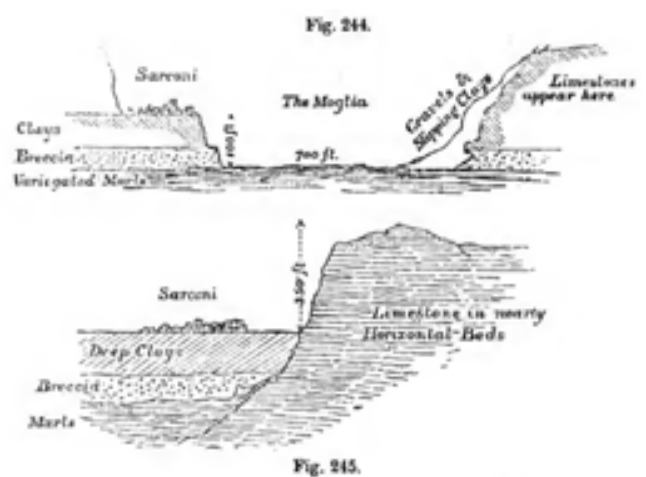

Fig. 9. Robert Mallet, Pianta e sezione del borgo di Sarconi (Mallet, Robert. 1862. Great Neapolitan earthquake of 1857. The first principles of observational seismology as developed in the report to the Royal Society of London of the expedition made by command of the Society into the interior of the Kingdom of Naples, to investigate the circumstances of the great earthquake of December 1857. London: Chapman and Hall, t.1, 414, figg. 244-245)

connesse alla lingua, nonché il suo interesse per molteplici discipline, compresa l'architettura.

Una parte rivelante del suo racconto corrisponde ai capitoli relativi al passaggio dello studioso nei centri urbani colpiti sia dal terremoto del 1851 sia da quello del $1857^{33}$.

Mallet per alcuni di questi paesi, come Melfi e Barile, si soffermò anche sull'elencazione dei danni prodotti dal sisma del 1851, fornendo qualche dato storico e di tipo geologico. Le sue descrizioni della città di Melfi, Rapolla, Barile, Rionero appaiono utili per sia per capire il livello di danneggiamento dei paesi sia per analizzare, attraverso gli esempi riportati, I'avanzamento della ricostruzione successiva al sisma del 1851. Seguendo la narrazione è possibile dedurre che la maggior parte dei centri del Vulture non avesse subito danni ingenti a causa del terremoto del 1857 anche perché molti edifici erano già stati completamente distrutti dal precedente sisma. Alle descrizioni sono allegate numerose foto dei centri urbani di Barile, Rionero, Rapolla e Muro Lucano che purtroppo mostrano solo una visione d'insieme dei borghi, rendendo difficile I'identificazione dei singoli monumenti ${ }^{34}$. Questi ultimi, tuttavia, vennero ben descritti nel testo. Nel caso di Rionero, ad esempio, definito come florido paese di fondazione albanese, la ricostruzione procedeva velocemente nonostante un terzo del bor- 


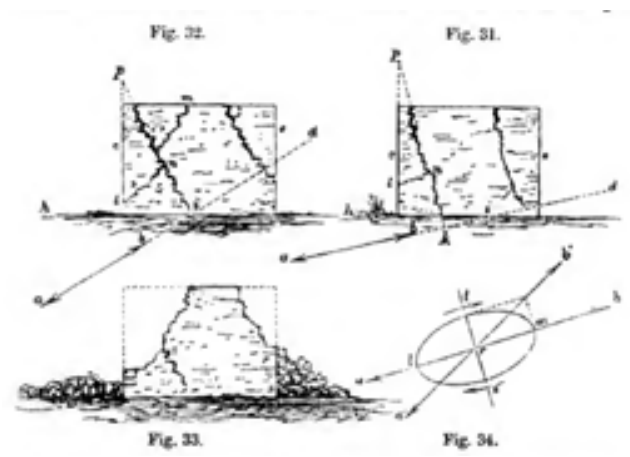

Fig. 10. Robert Mallet, Effetti dell'onda sismica su una muratura (Mallet, Robert. 1862. Great Neapolitan earthquake of 1857. The first principles of observational seismology as developed in the report to the Royal Society of London of the expedition made by command of the Society into the interior of the Kingdom of Naples, to investigate the circumstances of the great earthquake of December 1857. London: Chapman and Hall, t.1, 53, figg. 32-34)

go fosse stato distrutto dal sisma del Vulture. In molti palazzi, inoltre, erano già stati inseriti tiranti in ferro con funzione di consolidamento. Si tratta di informazioni davvero significative e utilissime per una possibile ricerca inerente la storia della ricostruzione e del restauro di tali centri urbani.

L'opera di Mallet venne conclusa con una riflessione interessante: I'autore si scusò con i lettori di non aver potuto inserire nella trattazione una sezione relativa ai metodi e ai sistemi costruttivi da impiegare nelle zone sismiche. Tale capitolo, a suo parere, sarebbe stato molto utile per l'Inghilterra che, nonostante il basso grado di sismicità, si trovava a capo di numerose colonie flagellate dai terremoti. La grande esperienza maturata nel corso del viaggio unitamente alle sue competenze professionali gli avrebbero permesso di fornire consigli sicuramente molto utili ${ }^{35}$. Si limitò, invece, semplicemente a ribadire ancora una volta come i danni causati dal terremoto sarebbero potuti essere di gran lunga inferiori se tutti gli edifici fossero stati costruiti con accuratezza utilizzando materiali di buona qualità ${ }^{36}$.
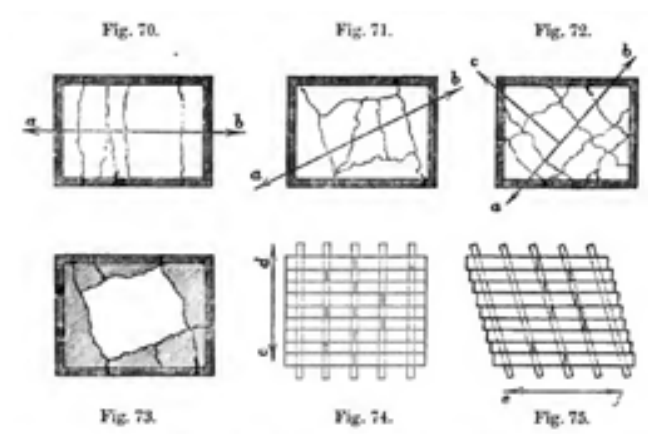

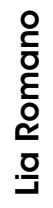

Fig. 11. Robert Mallet, Effetti dell'onda sismica sui solai (Mallet, Robert. 1862. Great Neapolitan earthquake of 1857. The first principles of observational seismology as developed in the report to the Royal Society of London of the expedition made by command of the Society into the interior of the Kingdom of Naples, to investigate the circumstances of the great earthquake of December 1857. London: Chapman and Hall, t.1, 103, figg. 70-75)

Appare deducibile come le descrizioni e i disegni oggetto del contributo risultino di grandissimo interesse per numerosi settori disciplinari anche molto distanti tra loro: la storia dell'arte, la storia della sismica, la geologia, l'architettura e la storia della costruzione. Per ognuno di tali ambiti i temi trattati offrono molteplici possibilità di approfondimento aprendo la strada a possibili ulteriori ricerche. Per quanto riguarda la storia delle tecniche costruttive e dell'architettura, discipline delle quali mi occupo, si può senza dubbio affermare che la documentazione iconografica e i testi associati risultano di grande ausilio per la definizione di una storia evolutiva e costruttiva dei centri urbani e degli edifici rappresentati, nonché di una storia del restauro. Lo sviluppo e l'intreccio di tali "storie" rappresenta uno strumento fondamentale non solo per lo studio e la comprensione di un territorio ad alto rischio sismico ma anche per la definizione di consapevoli interventi di restauro delle città e dei monumenti. Lo studio del passato può avere in tal modo ricadute immediate e pratiche sul nostro presente e sulla salvaguardia del patrimonio culturale. 
NOTE

1 Enzo Boschi, Emanuela Guidoboni and Graziano Ferrari, Catalogue of strong Italian earthquakes from $461 \mathrm{~b}$. C. to 1997 (Bologna: Editrice Compositori, 2000).

2 Reale Accademia delle Scienze e delle Belle Lettere di Napoli, Istoria de' Fenomeni del Tremoto avvenuto nelle Calabrie, e nel Valdemone nell'anno 1783, Atlante Iconografico (Napoli: Giuseppe Campo Impressore, 1784, 2 t.); Giovanni Vivenzio, Istoria dè tremuoti avvenuti nella Provincia di Calabria Ulteriore, e nella Città di Messina nell'anno 1783. (Napoli: Stamperia Reale, 1783); Giovanni Vivenzio, 1788. Istoria dè tremuoti avvenuti nella Provincia di Calabria Ulteriore, e nella Città di Messina nell'anno 1783 e di quanto nella Calabria fu fatto per il suo risorgimento fino al 1787 (Napoli: Stamperia Reale, 1788, t.2).

3 II regolamento è riportato integralmente come appendice della ristampa anastatica dell'opera di Giovanni Vivenzio a cura di Gregorio Rubino, cfr. Gregorio Rubino, Premessa, saggio introduttivo e schede nella ristampa della Istoria e teoria de' tremuoti in generale ed in particolare di quelli della Calabria, e di Messina del 1788, Atlante di Giovanni Vivenzio (Roma-Catanzaro: Giuditta Editore, 1993), 90-97.

4 Aloisio Antinori (ed.), Da contado a provincia. Città e architettura in Molise nell'Ottocento preunitario (Roma: Gangemi, 2006); Zullo, Enza (ed.). "Architettura e terremoto in Molise". Atti di convegno "Il Molise, il terremoto e la festa di S. Anna", 2 luglio 2005 (Campobasso: Palladino, 2009); Lia Romano, "Dentro la catastrofe. ॥ terremoto del 1805 tra emergenza e prima remissione dei danni a Napoli e in Terra di Lavoro", Storia urbana, no. 152-153 (luglio-dicembre 2016): 59-77; Lia Romano. "Catastrofe come lento mutamento. II terremoto del 1805 e le dinamiche di trasformazione del paesaggio e dell'architettura molisana". In Delli aspetti de paesi. Vecchi e nuovi Media per I'Immagine del Paesaggio, eds. Francesca Capano, Maria Ines Pascariello and Massimo Visone (Napoli: Cirice, 2016), 415-424; Lia Romano,
"Tra arte e scienza. Dal danno sismico alla sperimentazione di sistemi voltati 'leggeri' nel Regno delle Due Sicilie tra tardo Settecento e prima metà dell'Ottocento" (Phd diss. Università degli Studi di Napoli Federico II, capitoli 2 e 4), 41-97, 187-257).

5 Alfredo Buccaro (ed.), Potenza (Bari: Laterza, 1997); Giancarlo Boeri, Massimo Fiorentino and Piero Crociani, L'esercito borbonico dal 1830 al 1861 (Roma: Ufficio Storico SME, 1998,) 2 t.; Michael Rapport, 1848: I'anno della rivoluzione (Milano: Mondolibri, 2010).

6 Antonio Branno, et al. "Il terremoto del Vulture del 14 agosto 1851", Atti del 4 convegno annuale del gruppo nazionale di geofisica della terra solida (Roma: ESA, 1985): 329-336; Giuseppe Settembrino, "Il terremoto del 14 agosto 1851 nel distretto di Melfi". Radici, I (gennaio 1989): 115-128; Giuseppe Settembrino, "Per una storia della Cassa di prestanze agrarie e commerciali di Melfi istituita da Ferdinando II il 15 aprile 1852 (1852-1865, parte I)". Radici, n. 6: $113-124$.

7 Per approfondimenti sul terremoto cfr. Emanuela Guidoboni and Graziano Ferrari (eds.), Mallet's macroseismic survey on the Neapolitan earthquake of 16th december, 1857 (Bologna: SGA, 1987); Graziano Ferrari (ed.), Viaggio nelle aree del terremoto del 16 dicembre 1857. L'opera di Robert Mallet nel contesto scientifico e ambientale attuale del Vallo di Diano e della Val d'Agri (Bologna: SGA, 2004, t. 1); Graziano Ferrari (ed.), Ristampa anastatica e traduzione in italiano di // terremoto del 16 dicembre 1857. Primi principi di sismologia osservazionale sviluppati nel rapporto alla Royal society di Londra della spedizione condotta per conto della società all'interno del Regno di Napoli per studiare le circostanze del grande terremoto del dicembre 1857, di Robert Mallet (Bologna: SGA, 2004, t. 2); Graziano Ferrari (ed.), 16 dicembre 1857. Un grande disastro sismico. Da un terremoto laboratorio a laboratori sul territorio (Bologna: SGA, 2007, t. 3); Graziano Ferrari (ed.), Laboratorio Mallet. Fonti e studi su Robert Mallet e sui caratteri geodinamici dell' area lucana (Bologna: SGA, 2007, t. 4); Graziano Ferrari (ed.), Terremoti e paesaggio naturale e antropico. Previsione, preven- zione e sviluppo sostenibile (Bologna: SGA, 2009, t. 5); Graziano Ferrari (ed.), Viaggio nelle aree del terremoto del 16 dicembre 1857. Terremoti, storia e paesaggio (Bologna: SGA, 2009 t. 6); Iolanda Richichi, Implicazioni internazionali del terremoto lucano del 1857 (Firenze: Centro editoriale, 2011); Lia Romano. 2018. "Dalla catastrofe alla ricostruzione. Robert Mallet e il terremoto del 1857 in Basilicata tra disegno e fotografia", in La Città Altra. Storia e immagine della diversità urbana: luoghi e paesaggi dei privilegi e del benessere, dell'isolamento, del disagio, della multiculturalità, eds. Francesca Capano, Maria Ines Pascariello and Massimo Visone, 439-447. Napoli: CIRICE.

8 Si fa riferimento alle rappresentazioni di Nicola Palizzi, Salvatore Fergola e alle opere di Giacomo Paci, Relazione dei Tremuoti di Basilicata del 1851, (Napoli, Stab. Tipogr. Ministero dell'Interno, 1853); Luigi Palmieri and Arcangelo Scacchi, Della regione vulcanica del monte Vulture e del terremoto ivi avvenuto nel dì 14 agosto 1851 (Napoli: G. Nobile, 1852).

9 Antonio Lerra, "Il terremoto del 1857 in Basilicata. II ruolo delle istituzioni", Ricerche di storia sociale e religiosa, n. 25-26, (1984): 69-91.

10 Théophile Roller, Il governo borbonico innanzi alla coscienza dell'umanità ossia i provvedimenti del governo nella tremenda catastrofe del terremoto del 16 dicembre 1857. Notizie (Napoli, Giuseppe Marghieri, 1861).

11 Per approfondimenti sulla figura di Robert Mallet cfr. Ronald C. Cox (ed.), Robert Mallet, F.R.S., 1810-1881: Centenary Seminar Papers (Dublin: Institution of Engineers of Ireland, 1982). La pubblicazione a cui si fa riferimento nel testo è: Robert Mallet, Great Neapolitan earthquake of 1857 . The first principles of observational seismology as developed in the report to the Royal Society of London of the expedition made by command of the Society into the interior of the Kingdom of Naples, to investigate the circumstances of the great earthquake of December 1857 (London, Chapman and Hall, 1862). ॥ manoscritto originale di Robert Mallet unitamente al ricco corredo iconografi- 
co è conservato all'archivio della Royal Society di Londra (MS/224 e MS/225).

12 Floriana Conte (ed.), Impressione e verità nella pittura tra De Nittis, Patini e i Palizzi: dalla Puglia a Parigi attraverso la Via degli Abruzzi (Chieti: Edizioni Menabò, 2018).

13 Carla Fernández Martínez, "Imágenes de una catástrofe. El terremoto de Melfi de 1851 en la obra de Salvatore Fergola", Eikonocity, anno III, no. 1 (2018): 73-74. Carla Fernández Martínez, "Ciudad y terremoto. Algunos ejemplos de arquitectura antisísmica en la Edad Moderna", EDA. Esempi di Architettura, Settembre (2018): 1-15.

${ }^{14}$ Fernando Mazzocca, Luisa Martorelli and Antonio E. Denunzio (eds.), Fergola. Lo splendore di un regno, Catalogo della mostra (Napoli, 2 dicembre 2016 - 2 aprile 2017) (Venezia: Marsilio, 2016); Carla Fernández Martínez, "Imágenes de una catástrofe. El terremoto de Melfi de 1851 en la obra de Salvatore Fergola", Eikonocity, anno III, no. 1 (2018): 69-79.

15 Luigi Palmieri and Arcangelo Scacchi, Della regione vulcanica del monte Vulture e del terremoto ivi avvenuto nel dì 14 agosto 1851 (Napoli: G. Nobile, 1852).

16 Ivi, 131-1331.

17 Giacomo Paci, Relazione dei Tremuoti di Basilicata de 1851, (Napoli, Stab. Tipogr. Ministero dell'Interno, 1853).

18 Ivi, 87.

19 Va evidenziato che l'opera del Fergola alla quale ci riferiamo è nota come "La chiesa di Sant'Agostino parzialmente crollata nel terremoto di Melfi del 14 agosto 1851". La veduta, conservata al Museo Nazionale di San Martino, è stata erroneamente identificata con la chiesa di Sant'Agostino ma rappresenta, in realtà, il fronte danneggiato della cattedrale di Rapolla. Si ringrazia la dottoressa Carla Fernández Martínez dell'Università di Santiago de Compostela per aver identificato il soggetto e averne discusso con l'autrice dell'articolo. Si veda anche Fernando Mazzocca, Luisa Martorelli and Antonio E. Denunzio (eds.), Fergola. Lo splendore di un regno, Catalogo della mostra (Napoli, 2 dicembre 2016 - 2 aprile
2017) (Venezia: Marsilio, 2016), 182. Le opere di Achille Flauti e Francesco Palma sono contenute rispettivamente in allegato ai testi già citati di L. Palmieri, A. Scacchi e di Giacomo Paci.

${ }^{20}$ In particolare il sesto della volta rappresentata dal Flauti sembra più schiacciato di quello raffigurato dal Fergola e dal Paci.

${ }^{21}$ Fabio Speranza, "Alphonse Bernoud pioniere della fotografia. Luoghi, persone, eventi", in Alphonse Bernoud: pioniere della fotografi. Luoghi, persone, eventi, ed. Fabio Speranza (Napoli: Arte'm, 2018), 34-36.

22 Robert Mallet, Great Neapolitan earthquake of 1857. The first principles of observational seismology as developed in the report to the Royal Society of London of the expedition made by command of the Society into the interior of the Kingdom of Naples, to investigate the circumstances of the great earthquake of December 1857 (London, Chapman and Hall, 1862).

${ }^{23}$ Per approfondimenti si rimanda alla nota $\mathrm{n} .11$

${ }^{24}$ Mallet evidenziò come il nuovo metodo di indagine messo a punto non fosse stato utilizzato né in occasione del sisma del 1783 né in seguito del terremoto di Melfi del 1851. II tecnico irlandese scrisse di aver letto anche l'opera di Francesco Saverio Arabia, Relazione storica del tremuoto di Basilicata nell'anno 1851 letta nella tornata del 14 dicembre dell'Accademia Pontaniana dal socio residente Francesco Saverio Arabia (Napoli: dalla Stamperia del Vaglio, 1852).

25 Raffaele Battista, // terremoto di Basilicata. Relazione di Raffaele Battista segretario perpetuo della Real Societa economica di Basilicata (Potenza: dai torchi di Vincenzo Santanello, 1858); Nicola Carpinelli, Saggio filosofico sull'innormale movimento della terra applicato al tremuoto di Basilicata. Riflessioni del dottore Nicola Carpinelli (Napoli: Stabilimento Tipografico di V. Santanello, 1858); Luigi Maria Greco, Degli scrittori che han trattato dei tremuoti di Basilicata nel decimonono secolo. Memoria (Cosenza: G. Migliaccio, 1858); Giacomo Racioppi, Sui tremuoti di Basilicata nel dicembre 1857. Me- moria (Napoli: Stabilimento tipografico della Gazzetta dei Tribunali, 1858)

26 Soprintendenza generale agli interventi post-sismici in Campania e Basilicata, Dopo la polvere. Rapporto sui beni culturali a 10 anni dal terremoto (Roma: Associazione Mecenate 90, 1990); Fabrizio Terenzio Gizzi and Nicola Masini, Dalle fonti all'evento. Percorsi, strumenti e metodi per l'analisi del terremoto del 23 luglio 1930 nell'area del Vulture (Napoli: Edizioni scientifiche italiane, 2010).

27 Robert Mallet, Great Neapolitan earthquake of 1857. The first principles of observational seismology as developed in the report to the Royal Society of London of the expedition made by command of the Society into the interior of the Kingdom of Naples, to investigate the circumstances of the great earthquake of December 1857 (London, Chapman and Hall, 1862), t. 1, 414, fig. 245

$$
\begin{aligned}
& { }^{28} \text { Ivi, t. } 1,112 . \\
& { }^{29} \text { Ivi, t. 1, 325-326. } \\
& { }^{30} \text { Si cita a tal proposito la ricerca }
\end{aligned}
$$
condotta dal professore Francesco Doglioni dell'Universita IUAV di Venezia a seguito del sisma umbro-marchigiano del 1997, cfr. Francesco Doglioni (ed.), Codice di pratica (linee guida) per la progettazione degli interventi di riparazione, miglioramento sismico e restauro dei beni architettonici danneggiati dal terremoto umbro-marchigiano del 1997 (Ancona: Bollettino Ufficiale della Regione Marche, 2000).

31 Robert Mallet, Great Neapolitan earthquake of 1857. The first principles of observational seismology as developed in the report to the Royal Society of London of the expedition made by command of the Society into the interior of the Kingdom of Naples, to investigate the circumstances of the great earthquake of December 1857 (London, Chapman and Hall, 1862), t. 2, 67-71, chapter XXXIII "Conclusion of the examination of the city of Potenza", fig. 312

32 Ivi, t. 2, 73-78, chapter XXXIV "Signor d'Errico document - Calculation of the moment of shock at his station".

33 Ivi, t. 2, 83-116 (capitoli XXXVII-XLII della seconda parte). 
을 $\quad{ }^{34} \mathrm{Cfr}$. le fotografie allegate al ma-

들 noscritto di Mallet e conservate presso la

ह Royal Society di Londra (MS/225). Le foto

․ sono state pubblicate in Graziano Ferrari

으 (ed.), Viaggio nelle aree del terremoto del 16 dicembre 1857. L'opera di Robert Mallet nel contesto scientifico e ambientale attuale del Vallo di Diano e della Val d'Agri (Bologna: SGA, 2004, t. 1).

35 Mallet rimanda tale trattazione a una successiva pubblicazione che tuttavia non sembra essere stata realizzata. Alla fine del testo citò l'esempio dell'opera di Vincenzo Colosimo sul terremoto calabro del 1832 per le condizioni da osservare nella costruzione degli edifici in zona sismica, cfr. Vincenzo Colosimo, sul terremoto della Calabria avvenuto nella sera del dì 8 marzo 1832 (Napoli : dalla tipografia del Filiatre-Sebezio, 1832).
${ }^{36}$ Robert Mallet, Great Neapolitan earthquake of 1857. The first principles of observational seismology as developed in the report to the Royal Society of London of the expedition made by command of the Society into the interior of the Kingdom of Naples, to investigate the circumstances of the great earthquake of December 1857 (London, Chapman and Hall, 1862), t. 2, 384-386, capitol XXII "Retrospects and concluding words". 


\section{REFERENCES}

Antinori, Aloisio (ed.). 2006. Da contado a provincia. Città e architettura in Molise nell'Ottocento preunitario. Roma: Gangemi.

Arabia, Francesco S. 1852. Relazione storica del tremuoto di Basilicata nell'anno 1851 letta nella tornata del 14 dicembre dell'Accademia Pontaniana dal socio residente Francesco Saverio Arabia. Napoli: dalla Stamperia del Vaglio.

Battista, Raffaele. 1858. I/ terremoto di Basilicata. Relazione di Raffaele Battista segretario perpetuo della Real Società economica di Basilicata. Potenza: dai torchi di Vincenzo Santanello.

Boeri, Giancarlo, Fiorentino Massimo, and Piero Crociani. 1998. L' esercito borbonico dal 1830 al 1861. 2 t. Roma: Ufficio Storico SME.

Boschi Enzo, Guidoboni Emanuela, and Graziano Ferrari. 2000. Catalogue of strong Italian earthquakes from 461 b. C. to 1997. Bologna: Editrice Compositori.

Branno Antonio, Esposito Eliana, Luongo Giuseppe, Marturano Aldo, Porfido Sabina, and Vittorio Rinaldis. 1985. "Il terremoto del Vulture del 14 agosto 1851." Atti del 4 convegno annuale del gruppo nazionale di geofisica della terra solida, 329-336. Roma: ESA.

Buccaro, Alfredo, ed. 1997. Potenza. Bari: Laterza.

Carpinelli, Nicola. 1858. Saggio filosofico sull'innormale movimento della terra applicato al tremuoto di Basilicata. Riflessioni del dottore Nicola Carpinelli. Napoli: Stabilimento Tipografico di V. Santanello.

Colosimo, Vincenzo. 1832. Sul terremoto della Calabria avvenuto nella sera del dì 8 marzo 1832. Napoli: dalla tipografia del Filiatre-Sebezio.

Conte, Floriana, ed. 2018. Impressione e verità nella pittura tra De Nittis, Patini e i Palizzi: dalla Puglia a Parigi attraverso la Via degli Abruzzi. Chieti: Edizioni Menabò.

Cox, Ronald C., ed. 1982. Robert Mallet, F.R.S., 1810-1881: Centenary Seminar Papers, Dublin: Institution of Engineers of Ireland.
Doglioni, Francesco, ed. 2000. Codice di pratica (linee guida) per la progettazione degli interventi di riparazione, miglioramento sismico e restauro dei beni architettonici danneggiati dal terremoto umbro-marchigiano del 1997, Ancona: Bollettino Ufficiale della Regione Marche.

Fernández Martínez, Carla. 2018. "Imágenes de una catástrofe. El terremoto de Melfi de 1851 en la obra de Salvatore Fergola." Eikonocity III (1): 69-79.

Fernández Martínez, Carla. 2018. “Ciudad y terremoto. Algunos ejemplos de arquitectura antisísmica en la Edad Moderna." EDA. Esempi di Architettura (Settembre): 1-15.

Ferrari, Graziano, ed. 2004. Viaggio nelle aree del terremoto del 16 dicembre 1857. L'opera di Robert Mallet nel contesto scientifico e ambientale attuale del Vallo di Diano e della Val d'Agri. t. 1. Bologna: SGA.

Ferrari, Graziano, ed. 2004. Ristampa anastatica e traduzione in italiano di // terremoto del 16 dicembre 1857. Primi principi di sismologia osservazionale sviluppati nel rapporto alla Royal society di Londra della spedizione condotta per conto della società all'interno del Regno di Napoli per studiare le circostanze del grande terremoto del dicembre 1857, di Robert Mallet. t. 2. Bologna: SGA.

Ferrari, Graziano, ed. 2007. 16 dicembre 1857. Un grande disastro sismico. Da un terremoto laboratorio a laboratori sul territorio. t. 3. Bologna: SGA.

Ferrari, Graziano, ed. 2007. Laboratorio Mallet. Fonti e studi su Robert Mallet e sui caratteri geodinamici dell' area lucana. t. 4. Bologna: SGA.

Ferrari, Graziano, ed. 2009. Terremoti e paesaggio naturale e antropico. Previsione, prevenzione e sviluppo sostenibile. t. 5. Bologna: SGA.

Ferrari, Graziano, ed. 2009. Viaggio nelle aree del terremoto del 16 dicembre 1857. Terremoti, storia e paesaggio. t. 6. Bologna: SGA.

Gizzi, Fabrizio Terenzio, and Nicola Masini. 2010. Dalle fonti all'evento. Percorsi, strumenti e metodi per I'analisi del terremoto del 23 luglio 
1930 nell'area del Vulture. Napoli: Edizioni scientifiche italiane.

Greco, Luigi Maria. 1858. Degli scrittori che han trattato dei tremuoti di Basilicata nel decimonono secolo. Memoria. Cosenza: G. Migliaccio.

Guidoboni, Emanuela, and Graziano Ferrari, eds. 1987. Mallet's macroseismic survey on the Neapolitan earthquake of 16th december, 1857. Bologna: SGA. https://doi. org/10.1163/182539189x01274

Lerra, Antonio. 1984. "Il terremoto del 1857 in Basilicata. II ruolo delle istituzioni." Ricerche di storia sociale e religiosa 25-26: 69-91.

Mallet, Robert. 1862. Great Neapolitan earthquake of 1857. The first principles of observational seismology as developed in the report to the Royal Society of London of the expedition made by command of the Society into the interior of the Kingdom of Naples, to investigate the circumstances of the great earthquake of December 1857. London: Chapman and Hall. https://doi.org/10.1098/rspl.1859.0092

Mazzocca Fernando, Martorelli Luisa, and Antonio E. Denunzio, eds. 2016. Fergola. Lo splendore di un regno. Catalogo della mostra (Napoli, 2 dicembre 2016 - 2 aprile 2017). Venezia: Marsilio.

Paci, Giacomo. 1853. Relazione dei Tremuoti di Basilicata de 1851. Napoli: Stab. Tipogr. Ministero dell'Interno.

Palmieri, Luigi, and Arcangelo Scacchi. 1852. Della regione vulcanica del monte Vulture e del terremoto ivi avvenuto nel dì 14 agosto 1851. Napoli: G. Nobile.

Racioppi, Giacomo. 1858. Sui tremuoti di Basilicata nel dicembre 1857. Memoria. Napoli: Stabilimento tipografico della Gazzetta dei Tribunali.

Rapport, Michael. 2010. 1848: I'anno della rivoluzione. Milano: Mondolibri.

Reale Accademia delle Scienze e delle Belle Lettere di Napoli. 1784. Istoria de' Fenomeni del Tremoto avvenuto nelle Calabrie, e nel Valdemone nell'anno 1783, Atlante
Iconografico. 2 t. Napoli: Giuseppe Campo Impressore.

Richichi, Iolanda. 2011. Implicazioni internazionali del terremoto lucano del 1857. Firenze: Centro editoriale.

Roller, Théophile. 1861. I/ governo borbonico innanzi alla coscienza dell'umanità ossia i provvedimenti del governo nella tremenda catastrofe del terremoto del 16 dicembre 1857. Notizie, Napoli: Giuseppe Marghieri.

Romano, Lia. 2016. "Dentro la catastrofe. II terremoto del 1805 tra emergenza e prima remissione dei danni a Napoli e in Terra di Lavoro." Storia urbana 152-153 (luglio-dicembre): 59-77.

Romano, Lia. 2016. "Catastrofe come lento mutamento. II terremoto del 1805 e le dinamiche di trasformazione del paesaggio e dell'architettura molisana." In Delli aspetti de paesi. Vecchi e nuovi Media per I'Immagine del Paesaggio, edited by Francesca Capano, Maria Ines Pascariello and Massimo Visone, 415-424. Napoli: CIRICE.

Romano, Lia. 2018. "Dalla catastrofe alla ricostruzione. Robert Mallet e il terremoto del 1857 in Basilicata tra disegno e fotografia." In La Città Altra. Storia e immagine della diversità urbana: luoghi e paesaggi dei privilegi e del benessere, dell'isolamento, del disagio, della multiculturalità, edited by Francesca Capano, Maria Ines Pascariello and Massimo Visone, 439-447. Napoli: CIRICE.

Romano, Lia. 2018. "Tra arte e scienza. Dal danno sismico alla sperimentazione di sistemi voltati 'leggeri' nel Regno delle Due Sicilie tra tardo Settecento e prima metà dell'Ottocento." Phd diss. Università degli Studi di Napoli Federico II.

Rubino, Gregorio. 1993. Istoria e teoria de' tremuoti in generale ed in particolare di quelli della Calabria, e di Messina del 1788, Atlante, di Giovanni Vivenzio. Roma-Catanzaro: Giuditta Editore, 90-97.

Settembrino, Giuseppe. 1989. "Il terremoto del 14 agosto 1851 nel distretto di Melfi." Radici I (gennaio): 115-128.

Settembrino, Giuseppe. 1990. "Per una storia della Cassa di prestanze agrarie e commerciali 
di Melfi istituita da Ferdinando II il 15 aprile 1852 (1852-1865, parte I)." Radici 6: 113-124.

Soprintendenza generale agli interventi post-sismici in Campania e Basilicata, 1990. Dopo la po/vere. Rapporto sui beni culturali a 10 anni dal terremoto. Roma: Associazione Mecenate 90.

Speranza, Fabio. 2018. "Alphonse Bernoud pioniere della fotografia. Luoghi, persone, eventi." In Alphonse Bernoud: pioniere della fotografi. Luoghi, persone, eventi, edited by Fabio Speranza, 34-36. Napoli: Arte'm.
Vivenzio, Giovanni. 1783. Istoria dè tremuoti avvenuti nella Provincia di Calabria Ulteriore, e nella Città di Messina nell'anno 1783. Napoli: Stamperia Reale.

Vivenzio, Giovanni. 1788. Istoria dè tremuoti

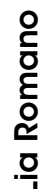
avvenuti nella Provincia di Calabria Ulteriore, e nella Città di Messina nell'anno 1783 e di quanto nella Calabria fu fatto per il suo risorgimento fino al 1787. t. 2. Napoli: Stamperia Reale.

Zullo, Enza, ed. 2009. "Architettura e terremoto in Molise." Atti di convegno I/ Molise, "Il terremoto e la festa di S. Anna", 2 luglio 2005. Campobasso: Palladino. 